\title{
Evaluation 2007
}

\section{Katholisch-Theologische Fakultät}

\author{
Gutachterbericht
}

\section{Stellungnahme der Fakultät zum Gutachterbericht}

\section{Gutachter:}

Prof. Dr. Walter Lesch

Prof. Dr. J ochen Martin

Prof. Dr. Hans Georg Ziebertz

Prof. Dr. Peter Hünermann

Prof. Dr. Ulrich Berges
UC Lourain

Albert-Ludwigs-Universität Freiburg

Universität Würzburg

Universtitä Tübingen

Universität Münster 
Prof. Dr. Peter Hünermann

Engwiesenstr. 14

72108 Rottenburg - Oberndorf

Tel. 07073/3725

e-mail: peter.huenermann@uni-tuebingen.de

Datei: Texte/Evaluation.Wien.117

\title{
Bericht über die Evaluation ${ }^{1}$ \\ der Katholisch-Theologischen Fakultät der Universität Wien vom 10.-14.11.2007
}

\author{
Kommission: Prof. em. Dr. Dr. h.c. mult. Peter Hünermann, Universität Tübingen \\ (Vorsitzender) \\ Prof. Dr. Ulrich Berges, Universität Münster \\ Prof. Dr. Walter Lesch, Université catholique de Louvain \\ Prof. Dr. em. Jochen Martin, Universität Freiburg i. Br. \\ Prof. Dr. Hans-Georg Ziebertz, Universität Würzburg
}

\section{Allgemeine Anmerkungen:}

Die Katholisch-Theologische Fakultät blickt auf eine seit dem Mittelalter ununterbrochene Geschichte in Forschung und Lehre zurück. Im deutschsprachigen Raum gehört sie zu den renommierten akademischen Ausbildungsstätten, nicht zuletzt wegen ihrer Einbettung in eine große Universität. In jüngerer Zeit fanden die pastoraltheologischen Untersuchungen ein breites europäisches Echo. Mit der Errichtung des Religionswissenschaftlichen Instituts und dem Institut für Spiritualität beschritt die Fakultät im Verlauf der zurückliegenden Jahrzehnte wichtige neue Wege. Viele Institute pflegen enge Kontakte und kooperieren seit Jahren mit anderen Universitätsinstituten bzw. Fakultäten.

Die jüngste Umstrukturierung der Universität stellt die Theologische Fakultät vor neue Herausforderungen. Die prägende Struktur der Katholisch-Theologischen Fakultät bilden die einzelnen Institute. Jeder Lehrstuhl bildet ein Institut, abgesehen vom Institut für Praktische Theologie (2 Lehrstühle).

Die Gliederung in übergreifende „Sektionen“ (biblisch-historische, praktische, systematische und Brückenfächer), wie sie sich der Evaluationskommission in den vorgeschlagenen und durchgeführten Gesprächsrunden darbot, ist eine formale Einteilung, die sich in dieser Gestalt nicht im schriftlichen Bericht selbst niederschlug. Sie markiert keine Arbeitseinheiten.

Zur Forschung:

a) Forschungsschwerpunkte

\footnotetext{
${ }^{1}$ Diese Evaluation stützt sich auf die zugesandten schriftlichen Unterlagen und Gespräche mit dem Rektorat, der Fakultätsleitung und den Mitgliedern der Institute.
} 
Zusätzlich zur bereits geübten Forschungstätigkeit wurde die Fakultät mit der Forderung konfrontiert, übergreifende Forschungsschwerpunkte (FSP) zu bestimmen. Dies hat bislang zu folgenden Resultaten geführt:

Das Institut für Praktische Theologie - aufbauend auf einer langjährigen interdisziplinären Arbeit und weitgehender, vor allem europäischer Vernetzung - verfolgt Pläne, die auf eine Forschungsplattform „Zentrum für Religionsforschung“ hinzielen. Parallel dazu hat sich ein FSP „Thesis“ zum „Dialog von Theologie, Ethik und Wissenschaften (Sciences) in der Gesellschaft“ herausgebildet, der eine großflächige Konsistenz anzudeuten scheint.

Für die anderen in der Selbstevaluation der Fakultät genannten FSP gilt, dass sie erste Ergebnisse eines Gespräches zwischen den Instituten bilden, die wesentlich vertieft und konkretisiert werden müssten. Es zeichnen sich durchaus transversale Linien ab, die sehr unterschiedliche theologische Disziplinen interessenmäßig untereinander verbinden. Zugleich müsste eine Konzentration der Themen und eine Vernetzung interdisziplinärer Art reflektiert werden.

Die Reduzierung und Fokussierung der FSP stellt die Fakultät vor die grundsätzliche Frage, ob sie eher fächerorientiert oder themenorientiert vorgehen will. Die Themenorientierung hätte den Vorteil einer transversalen Vernetzung, steht aber in der Gefahr, als reines „Hutprinzip“ wahrgenommen zu werden, das die fachspezifischen Fragen und Eigenheiten nicht genügend respektiert. Hier muss die Fakultät zu einer deutlicheren Zuordnung und Priorisierung ( und „Posteriorisierung“) kommen.

Es scheint der Kommission geboten, darauf hinzuweisen, dass die FSP in der Regel zeitlich auf etwa 5 Jahre begrenzt angelegt sein sollten, so dass auch zukünftige Neuorientierungen möglich bleiben. Die Publikationsleistungen und Lehrveranstaltungen sollten auch die FSP widerspiegeln, so dass Forschung, Forschungsergebnisse und Lehre eine größere Dichte erreichen.

Empfehlungen:

Die Kommission erlaubt sich, auf folgende Punkte hinzuweisen:

- am Anfang jeder Forschungsplanung muss eine genaue Fragestellung stehen;

- nächste Schritte betreffen die zu wählenden Methoden, die Vernetzung der entsprechenden Fachvertreter theologischer und nicht theologischer Disziplinen, die Programmierung der Forschung.

- Diese Schritte sind nicht möglich ohne die entsprechenden organisatorischen Entscheidungen: Bestellung eines Koordinators/Koordinationsgremiums, Bevollmächtigung durch die Interessenten, Bestimmung der Kommunikationswege etc.

- Analog zur Studienprogrammleitung könnte über ein eigenes Ressort „Forschungsprogrammleitung“ nachgedacht werden, das den Prozess der innerfakultären Abstimmung der Prioritäten hinsichtlich der FSP leitet, die Implementierung und Durchführung der FSP begleitet, die notwendigen internationalen Kontakte koordiniert und die Drittmitteleinwerbung auf nationaler und internationaler Ebene fördert.

- Bei der Fokussierung der FSP müssen die besondere Geschichte und Lage der Wiener Katholisch-Theologischen Fakultät mitberücksichtigt werden. Die Kernposition an der Schaltstelle von West- und Südosteuropa sollte ein wichtiges Element der 
Forschungsintensivierung darstellen. Hier liegt ein unbestreitbares „Alleinstellungsmerkmal“ der Fakultät. Bereits initiierte Projekte im „Donau-AlpenAdria-Raum“ könnten ins Gesamtbild integriert werden. Darüber hinaus sind die bereits in der Vergangenheit ausgebildeten interdisziplinären Kooperationen verschiedener Institute zu berücksichtigen.

- Bei zukünftigen Evaluationen sollten diejenigen Publikationen besonders markiert werden, die als Resultat der FSP angesehen werden. Ausdrücklich sollen monographische Publikationen hervorgehoben werden, um die Gefahr der Zerfaserung von Veröffentlichungen zu vermeiden und zu verdeutlichen, welche Ergebnisse die Forschungstätigkeit hatte und wo sich neue Felder und Perspektiven ergeben.

- Langfristig angelegte Forschungsplanungen bzw. weitreichende Forschungsperspektiven bedürfen einer Berücksichtigung bei Berufungsverfahren.

\section{b) Zur allgemeinen Forschungstätigkeit der Institute}

Jenseits der Frage nach FSP können die Bemühungen der Fakultät um Exzellenz nicht gelingen ohne eine herausragende Forschungstätigkeit der einzelnen Institute bzw. von Verbünden von Instituten, und - gegebenenfalls - interdisziplinärer Vernetzung mit nichttheologischen Disziplinen. Eine Forschungsprogrammleitung könnte auch in diesem Bereich hilfreich sein.

Hierzu einige Einzelbeobachtungen:

- Auffällig ist, dass trotz hohen Anteils der Lehre im AT und NT wenige Diplomarbeiten und Dissertationen entstehen. Erklärt wurde dies von den Fachvertretern mit den Sprachanforderungen. Zur Frage, ob eine in beiden genannten Fächern besonders standardisierte Lehre vielleicht weniger Anreize für die Studierenden biete, wurde entgegnet, dass in den Seminaren sehr wohl eigene spezielle Themen zur Bearbeitung kämen. Möglicherweise hänge dieser Mangel an Qualifikationsarbeiten auch mit dem relativ hohen Eintrittsalter der Studierenden zusammen: Sprachen lernen sich früh am leichtesten. Wenn die Disziplinen AT/NT/KG, die im Gespräch mit der Kommission unter „biblisch-historische“ Fächer gemeinsam angetreten waren, sich nicht zu gemeinsamen Themenschwerpunkten zusammenfinden können, ist dies vornehmlich auf divergierende methodologische Ausrichtungen zurückzuführen: Das AT (unter Leitung von Prof. Ludger Schwienhorst-Schönberger optierte für eine prononcierte pneumatologische Schriftauslegung (vgl. S. 40). Das Institut für NT tritt als Anwalt „historischer Theologie an der Fakultät“ (S. 58) auf. So fühlt sich das Institut für AT dem Themenschwerpunkt „Gottesfrage“ näher, während sich das NT auf die Frage der Mission in der Frühkirche konzentrieren will. Genese und Geltung, Wachstum und Bedeutung der einen Schrift von Altem und Neuem Testament, entstehungs- und rezeptionsgeschichtliche Fragestellungen, sollten aber nicht auf Dauer derart getrennt erforscht werden.

Für die internationale Sichtbarkeit sind auch in den biblischen Fächern regelmäßige englischsprachige Publikationen von wachsender Bedeutung, was es mehr als bisher zu berücksichtigen gilt.

- Das Institut für Kirchengeschichte hat im Rahmen der gesamten Geschichte der katholischen Kirche einen Forschungs- und Lehrschwerpunkt im Bereich kirchlicher und religiöser Alltagsgeschichte. So sehr der regionale Fokus auf den Donau-Alpen- 
Adriaraum zu beachten ist, bleiben doch Fragen: Gehört das Frintaneum-Projekt zu einer kirchlichen Alltagsgeschichte, und was leistet dies im Vergleich mit einer Alltagsgeschichte, wie sie am Wiener Institut für Sozialgeschichte betrieben wird? Wäre vielleicht eine Kooperation mit diesem Institut sinnvoll?

- Für den Bereich der Ethik ist die Fakultät mit zwei Instituten und ihren interdisziplinären Vernetzungen gut aufgestellt. Im Gespräch wurde die Frage erörtert, ob eine engere Kooperation zwischen Moraltheologie und Sozialethik ratsam sei, um das Fach in der universitären und außeruniversitären Öffentlichkeit sichtbarer zu machen. Die Bezeichnung „Moraltheologie“ wird in säkularer Umgebung oft als Stigma empfunden. An anderen Universitäten übliche Bezeichnungen wie „Theologische Ethik“ oder „Christliche Ethik“ könnten Abhilfe schaffen.

- Die Fakultät ist Mitträgerin und Gründungsmitglied des interfakultären Instituts für „Ethik und Recht der Medizin“, dort zurzeit aber nach unerfreulichen personellen Querelen nicht repräsentiert. Dieser Zustand ist nach Auffassung der Gutachter unbedingt zu korrigieren, um der Reputation der Fakultät nicht zu schaden, zumal gerade dieses Institut mit den qualifizierten Beiträgen aus der Katholischen Theologie das Potential zu einer international anerkannten Forschungs - und Beratungsplattform hat.

- Das Institut für Sozialethik hat mit seiner international beachteten Spezialisierung auf dem Gebiet der ökumenischen Sozialethik - unter besonderer Berücksichtigung der orthodoxen Kirchen und in Fortführung dieser Kontakte im Dialog mit dem Islam eine besondere Affinität zum Regionalschwerpunkt „Ost- und Südosteuropa“ der Universität Wien. Sowohl in diesen Forschungen als auch in bioethischen Beiträgen aus der Moraltheologie zeigen sich die gesellschaftspolitische Sensibilität und die europäische Dimension der Wiener Katholisch-Theologischen Fakultät, die im Wettbewerb um Drittmittel einschließlich der von der EU finanzierten Programme gute Chancen hat. Es ist von daher nicht recht nachvollziehbar, dass zurzeit Bemühungen um das Forschungsthema „Gerechtigkeit/Solidarität im Globalisierungszusammenhang“ ruhen.

- Die Institute für Religionswissenschaft und Christliche Philosophie erfüllen mit ihren Forschungs- und Lehrleistungen wichtige Brückenfunktionen, denen im heutigen gesellschaftlichen Kontext hohe Aufmerksamkeit zu schenken ist. Die religionswissenschaftliche Forschung ist fest im interfakultären Kontext verankert, wobei dem Institut eine koordinierende und inspirierende Rolle zukommt. Innovative Arbeiten zur Genderforschung und Religionsästhetik verdienen ebenso eine Erwähnung wie die Beteiligung an Konzepten einer islamischen Religionspädagogik und die renommierten Beiträge des Lehrstuhlinhabers zu Fragen der Religionskritik, insbesondere zur Nietzsche-Forschung.

- Die christliche Philosophie übernimmt eine wichtige Ausbildungsfunktion im Studium der Katholischen Theologie. Daraus erklärt sich die überdurchschnittliche Belastung der Mitarbeitenden in der Lehre, die sich leider nicht in der Wahl von Themen aus diesem Fachgebiet für Dissertationen auswirkt. Neben Standardthemen philosophischer Grundfragen in der Theologie hat sich ein Schwerpunkt in interkultureller Philosophie herauskristallisiert. Die am Institut beheimatete Chefredaktion der Zeitschrift „Polylog“ ist ein Aushängeschild dieser Forschungen mit internationaler Ausstrahlung. 
- Das Institut für Theologie und Geschichte des christlichen Ostens gehört zu den wenigen Instituten im deutschen Sprachraum mit einer solchen Ausrichtung und hat u. a. durch die enge Vernetzung mit Pro Oriente - eine Sonderstellung. Das Engagement des Instituts richtet sich vor allem auf den ökumenischen Prozess mit den Ostkirchen. Insbesondere soll deren Ausbildungskultur an den theologischen Lehranstalten untersucht werden. Das scheint - angesichts der bestehenden internationalen Kontakte - ein viel versprechender Weg zu sein.

- Das Institut für Fundamentaltheologie weist im Rahmen der Theologischen Fakultät die höchste Anzahl von abgeschlossenen Dissertationen und Diplomarbeiten auf. Es stellt den Koordinator für den angedachten FSP „Thesis“ und bemüht sich um einen interdisziplinären Forschungsverbund: Spiritualität und Gottesfrage heute. Das Institut pflegt zahlreiche Kontakte mit ausländischen, vornehmlich theologischen Institutionen und zählt auf Grund der persönlichen Forschungsschwerpunkte und der entsprechenden Publikationen seiner Professoren zu den bedeutendsten Fundamentaltheologischen Instituten Europas. Es stellt sich die Frage, ob eine gewisse Bündelung der Kräfte und Forschungsinteressen nicht zu einer wirkkräftigeren, Theologie überschreitenden Wahrnehmung führen würde.

- Die Fakultät besitzt - abweichend von anderen großen Katholischen Theologischen Fakultäten - nur einen Lehrstuhl für Dogmatik. Die Sakramententheologie ist allerdings in Forschung und Lehre an den Liturgiewissenschaftlichen Lehrstuhl gebunden. $\mathrm{Zu}$ berücksichtigen ist in diesem Kontext, dass im Bereich der Kirchengeschichte kein Lehrstuhl für alte Kirchengeschichte und Patrologie existiert. Die üblicherweise dort behandelten und zur Forschung anstehenden Themen stehen in einem engen Zusammenhang mit der Dogmatik, z. B. Konziliengeschichte etc. Die Patrologie wird vom Lehrstuhlinhaber für Ökumene wahrgenommen, der selbst kein ausgewiesener Fachvertreter in diesem Bereich ist. Soll hier im Bereich systematischer Theologie kein bedauerlicher Schwachpunkt in der Fakultät entstehen, müsste im anstehenden Berufungsverfahren darauf geachtet werden, dass ein Kandidat zum Zuge kommt, der - ausgerüstet mit den notwendigen philosophischen, theologie - und dogmengeschichtlichen Kenntnissen - die Interessen und Kräfte ganz auf wesentliche Fragen seines Faches konzentriert und nicht auf vielleicht verlockende Nebenfragen ausweicht.

- Empfohlen wird eine enge Kooperation in Forschung und Lehre zwischen dem Institut für Dogmatik, dem Institut für Liturgiewissenschaft (Sakramententheologie), Ökumene (Patrologie und Ostkirchenkunde) sowie dem Institut für Spiritualität und dem Institut für Fundamentaltheologie, um durch die Bündelung der Kräfte zu einer Forschungsqualität im weiten Bereich systematischer Theologie bzw. der Glaubenslehre zu kommen, welche der angestrebten Exzellenz der Fakultät entspricht.

- Die Perspektiven der Forschung im Institut für Liturgiewissenschaft sind weit gefächert, sie ergeben sich z. T. aus der starken Vernetzung mit US-amerikanischen Forschungsinstituten und der Filmakademie (vgl. Religiöse Rituale im Film). Es fällt auf, dass der Institutsleiter in den zurückliegenden Jahren zahlreiche Vorträge und Artikel zur armenischen Liturgie verfasst hat. Diese Thematik kommt in den Zukunftsplänen nicht mehr vor, obwohl in genereller Weise auf die Vorteile des Standortes Wien mit den vorzüglichen Anbindungen an den Osten hingewiesen wird. 
Die Forschungsthematik „Anglikanische Ordinationsriten“ sollte abgerundet und abgeschlossen werden. Insgesamt wären eine Konzentration der Themen und ihre zügige Aufarbeitung empfehlenswert, ebenso wie eine stärkere Vernetzung mit den benachbarten theologischen Disziplinen.

- Das Institut für Kirchenrecht pflegt eine sehr starke Kooperation mit dem bedeutenden kirchenrechtlichen Institut der Theologischen Fakultät in Lugano. Der Institutsleiter ist dort seit 2001 „ständiger Gastprofessor“. Ferner arbeitet das Institut eng mit der juristischen Fakultät zusammen. Die Kooperation mit Fächern der eigenen Fakultät erscheint - abgesehen von der Kooperation mit der Praktischen Theologie - nicht so ausgeprägt. Die Kommission legt nahe - neben der Erarbeitung eines Lehrbuches für Verfahrensrecht und Sanktionen - eine auch forschungsmäßige Beschäftigung mit dem orientalischen und generell dem ökumenischen Kirchenrecht (vgl. Rechtssprache des CCEO) - in Absprache mit dem Institut für Theologie und Geschichte des christlichen Ostens - zu realisieren.

- Der Zusammenschluss der Institute Religionspädagogik und Pastoraltheologie zu einem Institut für Praktische Theologie ist zu begrüßen. Ein Entwicklungsplan als Umsetzung des bereits erarbeiteten Leitbildes sollte möglichst bald in Angriff genommen werden. Ein interfakultäres Zentrum für Religionsforschung könnte das an der Fakultät vorhandene Potential mit jenem der anderen Fakultäten so bündeln, dass eine spezifische Exzellenz erreichbar sein sollte.

Die Leistungen des Arbeitsbereichs Pastoraltheologie und Kerygmatik sind im Bereich Zentral- und Osteuropa herausragend, doch seine Brückenfunktion könnte Wien noch besser erfüllen, wenn man auch im englischsprachigen Raum präsenter wäre. Das schließt ein: stärkere Teilnahme am fachwissenschaftlichen Diskurs auf internationalen Tagungen, Publikationen in englischsprachigen Journalen etc.

Der Arbeitsbereich Religionspädagogik und Katechetik war - bedingt durch das Ausmaß der Pflichtlehre und die vorhandenen Ressourcen - stark an die Lehre gebunden. Dies ist zwar für innovative Lehr- und Lernformen genutzt worden (Lernwerkstatt, eLearning, Blended Learning, ePortfolio,...), die Forschung blieb dahinter allerdings zurück. Der Umbau in Richtung einer stärkeren Forschungsorientierung ist sicherzustellen.

Die geplante Gründung eines Fachdidaktikzentrums für Katholische Religion ist zu befürworten, womit zugleich ein Forschungskonzept verbunden sein sollte. Das Arbeitsgebiet „Schulentwicklung und Religion“ ist im religionspädagogischen Diskurs singulär und kann in Verbindung mit entsprechend qualifizierten Forschungsarbeiten zur Profilbildung beitragen. Hervorzuheben ist, dass das Institut für das Forschungsnetzwerk REVACERN mit einem sechsstelligen Betrag von der EU gefördert wird. Insgesamt gilt, dass ein großer Teil der geleisteten Forschungsarbeit universitär nicht sichtbar ist, weil die dafür angeworbenen Drittmittel von der Universität nicht entsprechend eingeschätzt worden sind.

Zur Lehre:

Die relativ hohe Zahl von Studierenden an der Katholisch-Theologischen Fakultät Wien ist ein generelles Indiz für eine attraktive Lehrstruktur. 
Zu einer Reihe von Einzelproblemen:

- Die SPL geht sehr engagiert in der Planung der neuen Studienstruktur (Bachelor/Master) vor. Es ist dringend geboten, die Modulstruktur und die jeweilige Fächerzuordnung so zu regeln, dass die von Bologna gewünschte Mobilität der Studierenden nicht behindert, sondern gefördert wird. Die weithin in KatholischTheologischen Fakultäten des deutschen Sprachraums geübte Praxis, die Studierenden zu zwei „Auswärts“- bzw. „Auslands“- Semestern zu veranlassen, sollte gefördert werden Es ist ausdrücklich zu begrüßen, wenn sich einige Module, vornehmlich im Bachelor-Bereich, fachspezifisch, andere, vornehmlich im Masterbereich, themenorientiert zuordnen. So ergibt sich eine intelligente Verbindung zur fächer- und themenorientierten Forschung. In einigen Modulen könnte sich die Forschung direkt niederschlagen.

- Die Kommission macht das Rektorat darauf aufmerksam, dass auch nicht-staatliche, kirchliche europäische Universitäten und Fakultäten, wenn sie einen anerkannten hohen akademischen Standard aufweisen, in europäische Exchange-Programme aufgenommen werden sollten.

- Die Ansätze im e-learning sind viel versprechend, was nicht zuletzt vom Studienvertreter bestätigt wird. Die im e-learning angelegten Weiterbildungsmöglichkeiten sollten ausgebaut werden. Ein nach Südosteuropa ausgelegtes Fernstudium sollte im Auge behalten werden. Die Wiener Fakultät würde sich hier ein breites Feld reservieren, das an Bedeutung nur zunehmen kann.

- Das durchschnittliche hohe Einstiegsalter der Diplomstudierenden (30 Jahre) ist überraschend und lässt die Frage aufkommen, welche Berufsperspektiven sich für viele der „alten“ Studierenden nach Abschluss des 10-12semestrigen Studiums ergeben.

- Die nicht geringe Abbrecher-Quote ist nach Auskunft nicht zuletzt darauf zurückzuführen, dass die Diplomarbeit für viele ein großes Hindernis darstellt - auch für solche, die ansonsten befriedigende Leistungen zeigen. Durch die Verpflichtung zu Proseminaren und Seminaren mit Hausarbeiten soll diese „Schreibhürde“ abgebaut werden. Auch hier würden sich die FSP und Themenschwerpunkte gut einbauen lassen, wenn Diplom-Arbeiten gezielt überschaubare Aspekte der Forschungsinitiativen in Angriff nehmen würden. Zudem wäre so ein Schritt hin zum Aufbau von Graduiertenkollegs getan.

- Die Fakultät sollte der Frage von Doktoratsprogrammen besondere Aufmerksamkeit widmen. Ansätze solcher Programme werden im Institut für Fundamentaltheologie erprobt.

$\mathrm{Zu}$ den disziplinübergreifenden Organisations- und Lehrformen des Doktorandenstudiums wird empfohlen, auf die Erfahrungen mit Graduiertenkollegs und deren Weiterentwicklungen in fakultäts- bzw. universitätseigener Regie im deutschen Sprachraum zurückzugreifen. (Vgl. z. B. die „Graduiertenakademie“ im Rahmen der Universität Freiburg i. Br. etc.)

- Die Reorganisation der Doktorats- und Postdoktoratsphase (Habilitationsphase) führt zu der Frage, wie Jungwissenschaftler die nötigen Erfahrungen und Horizonterweiterungen in ausländischen wissenschaftlichen Einrichtungen machen 
können. Die Kommission empfiehlt, diesem Aspekt besondere Aufmerksamkeit zu widmen.

\section{Wissenschaftsorganisatorische Fragen:}

- In der Eingangsunterredung mit der Vizerektorin Frau Prof. Schnabl wurde darauf hingewiesen, dass die Universität eine Leitungs- und Managements-Struktur habe, was eine Stärkung des Rektorats und der Dekane impliziert. Wir erlauben uns, darauf hinzuweisen, dass diese Entwicklung auch Gefahren enthält. Zwar ist ein modernes Management für die Universität unerlässlich. Es ist allerdings ebenso unerlässlich, dass die Wissenschaftlerinnen, die die Forschung und Lehre tragen, zu einem frühen Zeitpunkt in die Entscheidungsprozesse eingebunden werden. Die Planung und Entwicklung neuer Forschungs- und Lehrstrukturen bedarf größerer Zeiträume und Vorlaufzeiten. Dies ist eine Problematik, auf die wir bei der Diskussion um die FSP gestoßen sind.

- Insgesamt sind die Universität Wien und die Katholisch-Theologische Fakultät sehr stark auf Drittmittel und deren Einwerbung angewiesen. Eine theologische Fakultät hat traditionell andere Zugänge zu Ressourcen als Betriebswirtschaftslehren oder andere Disziplinen. Es dürfte widersinnig sein, Drittmittel aus solchen spezifischen Ressourcen als „schlechte“ Drittmittel zu kategorisieren. Im Leistungskatalog der Fakultät soll zukünftig erscheinen, welche Vielzahl von Mitteln eingeworben wurden, welche Projekte unterstützt werden konnten und welche Forschungsergebnisse sie erbracht haben. Dass kirchliche Mittel fließen, ist zu begrüßen, weil dies zeigt, dass die Fakultät in einem zukünftigen Praxisfeld ihrer Studierenden relevante Forschung leistet.

- Das Drittmittelaufkommen an der Fakultät verteilt sich sehr unterschiedlich auf die Lehrstühle/Institute. Es gibt Institute mit einer hohen Summe an eingeworbenen Forschungsmitteln, während andere im Berichtszeitraum keinen Mitteleingang verzeichnen können. Drittmittel sind immer auch ein Zeichen für unterstützungsrelevante Forschung. Daher sollte die Fakultätsleitung im Benehmen mit den Betroffenen Maßnahmen entwickeln, wie Institute in die Lage versetzt werden können, Forschung stärker honorieren zu lassen. Diese Überlegungen hängen mit der Einrichtung möglicher Forschungsverbünde zusammen.

- In der Selbstevaluation wendet die Fakultät das ABC-Prinzip zur Taxierung der Veröffentlichungen in wissenschaftliche, fachliche und populärwissenschaftliche an. Unsicherheit besteht in der Definition der 3 Kategorien. Ein Bezugspunkt ist das sog. „Grimm-Papier“, das gegenüber der naturwissenschaftlichen Publikationspraxis der Eigenart der Geisteswissenschaften gerecht werden will. Die Listung der Veröffentlichungen an der Fakultät zeigt erhebliche Unterschiede im Verständnis. (Vgl. den Ausweis von Rezensionen, Beiträgen von 2-3 Seiten als A-Publikationen). Wegen der europäischen und internationalen Vergleichbarkeit empfiehlt die Kommission: die Überlegungen aus dem Grimm-Papier mit den Richtlinien der ESFHumanities abzugleichen und sich im Zweifel an den ESF-Standards zu orientieren; Ausnahmen im Blick auf bedeutende Fachjournale oder Reihen festzustellen; das wissenschaftliche Personal mit den Standards bekannt zu machen; fakultätsintern abzusprechen, welche Verteilung zwischen ABC-Publikationen in den einzelnen Instituten sinnvoll ist. 
- Wir geben die Anregung, dass die Katholisch-Theologische Fakultät ihre seit 1963 eingeschlafene Publikationsreihe „Wiener Theologische Studien“, die ein hohes Ansehen hatte, wieder aufleben lässt, zumal die Fakultät nicht über ein eigenes Fachjournal verfügt. Erforderlich dafür ist ein entsprechendes Konzept.

- Die Restrukturierung der Curricula im Bologna-Prozess hat u.a. das Ziel, eine Mobilität der Studierenden zu erleichtern und die Vergleichbarkeit der Studien auf europäischer Ebene zu steigern. Erhebliche Schwierigkeiten ergeben sich hier faktisch hinsichtlich der Anrechnungen erbrachter Studienleistungen von ausländischen Studierenden und dem Wechsel von Wiener Studierenden ins Ausland. Wir empfehlen dem Rektorat wie der Fakultät dringend, auf abgestimmte europäische Rahmenbedingungen für die Anrechnung zu drängen. Die Fakultäten werden sonst leicht überfordert.

- Die Wiener Theologische Fakultät umfasst zwei Ebenen: Dekanat/Fakultätsleitung und Lehrstühle (Institute). Dies ist eine Besonderheit im deutschsprachigen Raum, wo üblicherweise eine Zwischenstruktur von Instituten mit mehreren Lehrstühlen oder vereinigten Seminaren besteht. Es ist auch eine Besonderheit gegenüber dem angloamerikanischen Raum, wo in der Regel eine Einteilung in Departments vorhanden ist. Es stellt sich die Frage, ob die Organisationsebene der einzelnen Lehrstühle auf Dauer den wachsenden Anforderungen hinsichtlich einer Kooperation in Forschung und Lehre genügen kann. Hinzu kommt, dass durch den Wegfall der bisherigen „Pragmatisierungsregeln“ der Anteil an längerfristig beschäftigten jüngeren Wissenschaftlern in den einzelnen Instituten abschmelzen wird.

Im Übrigen zeigt sich, dass der Zusammenschluss der Fächer Religionspädagogik und Pastoraltheologie erfolgreich ist. Die Kommission empfiehlt, diese Frage behutsam und in einem längeren Prozess - im Zusammenhang mit den Klärungen von Forschungsschwerpunkten und der Reflexion auf die übrigen Forschungsthemen wie die Vernetzung der Disziplinen - zu erörtern und praktikable Organisationsformen herauszubilden. 


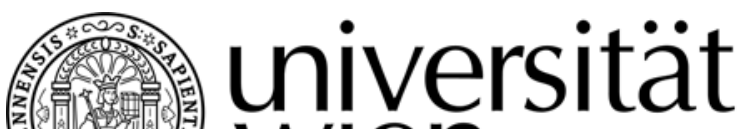 wien
}

Katholisch-Theologische Fakultät

Universität Wien

Qualitätssicherung

Maria-Theresien-Straße 3/15a

1090 Wien
Dekanat der Katholisch-Theologischen

Fakultät

Univ.-Prof. Dr. Martin J äggle

Dr.-Karl-Lueger-Ring 1

A- 1010 Wien

$\mathrm{T}+43$ (1) 4277-300 10

$\mathrm{F}+43$ (1) 4277-93 00

$\mathrm{M}+43-664-60277-30010$

martin.jaeggle@univie.ac.at

http:// www.univie.ac.at/ ktf/

Wien, am 06. Februar 2008

\section{Bericht über die Evaluation der Katholisch-Theologischen Fakultät der Universität Wien vom 10.-14.11.2007}

\author{
Stellungnahme der Katholisch-Theologischen Fakultät
}

\section{Allgemeines zur Evaluierung}

Die Fakultätsleitung der Katholisch-Theologischen Fakultät der Universität Wien begrüßt den gründlichen und umfassenden Evaluationsbericht, der für die weitere Entwicklung der Fakultät von großer Bedeutung ist. Viele der Empfehlungen, Würdigungen und Beobachtungen sprechen für sich (namentlich in der Lehre und der Wahrnehmung der meisten Institute) und können unmittelbar umgesetzt werden, sodass sich die Stellungnahme v.a. auf einzelne, nichts desto weniger entscheidende Empfehlungen beziehen wird.

Die positive Würdigung der Leistungen im Bereich der Lehre nimmt die Fakultät erfreut zur Kenntnis

\section{Forschung}

Von besonderer Tragweite ist die nachdrückliche Empfehlung der Evaluatoren, die Forschungsleistungen der Fakultät zu bündeln und neben der international angesehenen Forschung an den einzelnen Instituten und Fachbereichen eine nach außen wahrnehmbare gesamtfakultäre Forschung stärker sichtbar zu machen und generell die Forschung auch instituts- und fächerübergreifend zu profilieren. In diesem Kontext schlagen die Evaluatoren die Einrichtung eines „Forschungsdekans“" vor. Wir werden demgegenüber die bisherige Zuständigkeit des/der Vizedekan/in für die Forschung stärker profilieren. Derzeit wird eine Strategie ausgearbeitet, die hinkünftig eine gesamtfakultäre und institutsübergreifende sowie auch innerhalb der Wiener Universität vernetzte Forschung gewährleistet. Dies soll sich auch in einer verstärkten Einwerbung von Drittmittel niederschlagen. In Ausarbeitung ist u.a. ein gesamtfakultärer Forschungsschwerpunkt in Richtung „Religionsforschung“, an dem sich alle Institute gleichberechtigt beteiligen können und sollen und eine strukturelle Stärkung und Fokussierung der einzelnen fakultären Forschungsschwerpunkte. Ziel dabei ist es sowohl der Fakultät ein international wahrnehmbares Forschungsprofil zu geben als auch die theologische Kompetenz fakultätsübergreifend in die Universität Wien einzubringen. Dem spezifischen Standort Wien soll dabei Rechnung getragen werden. Ein besonderer Ansprechpartner ist die Evangelisch-Theologische Fakultät, mit der in Hinkunft unter Einbeziehung der beiden SABs (Scientific Advisory Boards) noch enger kooperiert werden soll. Besonderes Augenmerk will die Fakultät auf die Doktoratsausbildung legen, um Forschung und Lehre besser zu vernetzen und den wissenschaftlichen Nachwuchs disziplinen- und fakultätsübergreifend ausbilden und einbinden zu können. 
Um der Notwendigkeit einer verstärkten Außenwahrnehmung als Fakultät Rechnung zu tragen, will die Leitung den Vorschlag der Evaluatoren aufgreifen und ein Publikationsorgan einrichten, wobei Bezeichnung und Profil noch zu klären sind.

Was die Bewertung von Publikationen betrifft, nimmt die Fakultät am gesamtuniversitären Klärungsprozess teil. Der Empfehlung, eine umfassende Sondierung wissenschaftlicher Fachjournale - unter Einschluss theologieverwandter Disziplinen - vorzunehmen, um Publikationen in Hinblick noch gezielter platzieren zu können, wird Rechnung getragen und ein entsprechendes Verzeichnis an für die Theologie interessanten J ournalen und Reihen erstellt. In diesem Kontext wird die Fakultät eine Stellungnahme zur Revision des „Ranking of theological reviews" der „European Science Foundation" vornehmen.

Die Fakultät unterstreicht außerdem den Hinweis der Evaluatoren, dass die Reputation der Katholisch-Theologischen Fakultät im Bereich des Dialogs mit der Medizin dadurch geschwächt wird, dass ihre Beiträge aufgrund ihrer mangelnden offiziellen Präsenz nicht in angemessener Weise in die Forschungsplattform "Ethik und Recht in der Medizin" einfließen können.

\section{Stellungnahme einzelner Institute}

Von den meisten Instituten erhielt die Fakultätsleitung eine dezidiert positive Rückmeldung auf die Außenwahrnehmung der Fakultät und der einzelnen Fachbereiche, wie sie im vorliegenden Evaluationsbericht dargelegt wird. Von einigen Instituten liegen Reaktionen auf einzelne Anregungen und Forderungen vor, die hier zusammengefasst wiedergegeben werden sollen. Im Bereich der Bibelwissenschaften besteht die Absicht, über die bereits gegebene Zusammenarbeit hinaus enger zu kooperieren, die verschiedenen methodischen Zugänge zu diskutieren und mehr Studierende für die Abfassung von Diplomarbeiten und Dissertationen zu gewinnen. Bisherige Hürden durch die mangelnde Kenntnis der biblischen Sprachen - namentlich des Hebräischen - werden durch die neuen Studienpläne deutlich verringert. Verstärkt soll auch in englischer Sprache publiziert werden, allerdings gibt es mehrfach an der Fakultät - namentlich auch seitens des Instituts für kanonisches Recht - den Hinweis, dass Deutsch im Bereich der Theologie nach wie vor eine führende Wissenschaftssprache ist und bleiben wird. Das Institut für Kirchengeschichte macht darauf aufmerksam, dass durch die Neubesetzung der Professur auch das „Gesamtdesign“ des Instituts neue Impulse erfahren wird. Weiterhin bleibt der langfristig verfolgte Forschungsschwerpunkt einer "Kirchlichen Sozial- und Religiösen Alltagsgeschichte" (Kirchlich-religiöse Aspekte der Frau-Mann Relation während des Mittelalters, Rhetorik des spätmittelalterlichen Papsttums, Ars moriendi) sowie das internationale "Frintaneum-Projekt" oder das jüngere nationale Projekt "Pius XI. und Österreich". Im Bereich der Liturgiewissenschaft wird die Anregung der Evaluatoren aufgegriffen und die armenische Liturgie weiter in ökumenischer Perspektive erforscht werden. Ebenso soll aber auch das Forschungsprojekt „Anglikanische Ordinationsriten“ vorangetrieben werden. Das Institut für kanonisches Recht sieht die Notwendigkeit, künftige Evaluierungen auch auf das Dekanat zu beziehen. Es legt Wert darauf, festzuhalten, dass aktuell eine Kooperation mit den Instituten für Religionswissenschaft sowie Liturgiewissenschaft stattfindet. Das Institut für Praktische Theologie verweist auf die bereits vorhandene Einbindung in den westeuropäischen Raum z.B. durch die Teilnahme an der Europäischen Wertestudie. Im Blick auf die sicher zu berücksichtigende Urgenz nach verstärkter Präsenz im englischsprachigen Raum wird darauf hingewiesen, dass sich die Pastoraltheologie dort stark auf den Bereich der „pastoral care“ konzentriert, d.h. sie ist primär am Individuum und an poimenischen Fragen ausgerichtet; demgegenüber hat die Wiener Pastoraltheologie eine stark (religions-)soziologische und gesellschaftspolitische Ausrichtung. Der Umbau im Bereich der Religionspädagogik und Katechetik zugunsten der Forschung ist durch die Knappheit an Personal und die mehrjährige Leitungsverpflichtung des Ordinarius wesentlich behindert. Der geforderten verstärkten universitären Sichtbarkeit der umfangreichen durch Drittmittel finanzierten Forschung wird Rechnung getragen werden.

\section{Organisation}

Der Diskussionsprozess über die Organisationsstruktur der Fakultät wird fortgesetzt werden unter besonderer Berücksichtigung des Kriteriums der Stärkung der institutsübergreifenden Forschung und der Außenwahrnehmung. Dabei wird zu überprüfen sein, ob die derzeitige, fast ausschließliche Gliederung nach Lehrstühlen den diesbezüglichen Anforderungen adäquat Rechnung trägt. Die von den Evaluatoren angeregte institutionelle Kooperation der einzelnen systematischen Fächer soll im Rahmen der zu diskutierenden Architektonik der Fakultät beachtet werden. 
Zur Förderung von J ungwissenschafter/innen sollte geprüft werden, im Rahmen der Universität neue Möglichkeiten für Anstellungs- und Beschäftigungsformen zu finden (,tenure track").

$$
\text { Mut. Tial }
$$

Univ.-Prof. Dr. Martin Jäggle, Dekan 